\title{
Shuffle Exchange Network in Multistage Interconnection Network: A Review and Challenges
}

\author{
Nur Arzilawati Md Yunus and Mohamed Othman
}

\begin{abstract}
Multistage Interconnection Networks (MINs) are design to provide an effective communication in switching. MINs networks consist of stages that can route the switching through the path. In this types of network the major problem occur when the switch failed to route in the stage. If these situations occur the switching need to be route to an alternative path to avoid from system failure. Shuffle-exchange networks have been widely considered as practical interconnection systems due to their size of it switching elements and uncomplicated configuration. It can helps in fault tolerance and reduce the latency. As an illustration, there are mainly six types of SEN which are important to MINs, namely SEN with additional stages, Improved Irregular Augmented Shuffle Multistage Interconnection Network (IIASN), Irregular Modified Alpha Network (ALN), Irregular Augmented Shuffle Network (IASN), Irregular Augmented Shuffle Exchange Network (IASEN) and Generalized Shuffle Exchange Network (GSEN) were reviewed and described their challenges in this paper.
\end{abstract}

Index Terms-Multistage interconnection network, Shuffle exchange network, Additional stages, Fault tolerance, Switching element.

\section{INTRODUCTION}

In the past decade, MIN was applied in fields such as telecommunication and parallel computing. A parallel microprocessor system plays the mechanism needed for transferring information between processor and memory module, namely the interconnection network. Interconnection networks offer an attractive and economical solution to the communication and interconnection between system components [7]. MIN is known as cost efficient means to offer programmable data paths among functional modules in multiprocessor systems [2]. By adding Shuffle-Exchange Networks (SENs) in MIN it has been commonly consider as convenient interconnection systems due to their size of its switching elements (SEs) and uncomplicated configuration. SEN is a unique path it has only a single path between a particular input and output [4]. These networks are frequently applied with simple modular switches, make use of two input and two output switching elements [6]. The shuffle exchange multistage interconnection network is one network in large class topologically equivalent MINs that include the omega, indirect binary, cube, baseline, and generalized cube [9]. This network also can be implementing in different types of network sizes. The number of stages and the connection

Manuscript received February 10, 2011; revised October 11, 2011.

The authors are with the Department of Communication Technology and Network at Universiti Putra Malaysia. patterns between stages determine the routing capability of the networks [13]. A major problem in SEN is path complexity occur when the routing procedure have been apply. A critical set of components is defined as set of switching component, each from different groups, such as that a network a network failure will occur if all the components become faulty simultaneously [3]. SEN provide more redundant paths, by adding stage to the SEN. This network allows two paths for communication between each source and destination. SENs in addition designed to provide inter process communication and developed fault tolerant architecture. SENs functionality also can reduce the cost and latency in the interconnection process.

\section{Shuffle ExChange Network In Multistage INTERCONNECTION NETWORK}

SEN is a unique path it has only a single path between a particular input and output [9]. In MIN the routing of a message from given source to a given destination is based on the destination address [15]. The failure of a component in the interconnection network can bring down the entire system [15]. The interconnection among switches, number of switches and types of switches normally determine the system performance. MIN connects input devices to output devices through a number of switch stages. Reliability and performance of interconnection network system significantly depends on the interconnection of its components [9]. In general, multiple path MINs have higher network reliability compared to the single path MINs [7]. Shuffle-exchange networks have been widely considered as practical interconnection systems due to their size of its switching elements and uncomplicated configuration. Most of the researcher said that shuffle exchange network suitable in MIN architecture because it can provide an alternative path for routing procedure. Figure 1 shows example of SEN with 8x8 network size in Omega networks.

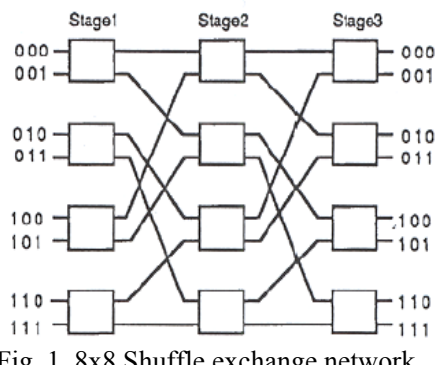

Fig. 1. 8x8 Shuffle exchange network

\section{Problem OVERVIEW}

Multistage Interconnection Networks are designed to provide an effective communication in switching [8]. By 
using SENs in MINs the stages can route the switching element through the path. Shuffle-exchange networks have been widely considered as practical interconnection systems due to their size of its SEs and uncomplicated configuration [5]. In this types of network the major problem arise when the switching element failed to route in the stages. To overcome this problem the switching element requires being route to an alternative path to avoid from system failure. By adding stages and routing procedure in SENs the switches is able to route to an alternative path. In addition, it also can improve the reliability of the network performance. SEN function is capable to provide inter process communication and developed fault tolerant architecture. These networks also provide permutation to route source to destination when both switches in a loop are simultaneously faulty at any stages [11].

\section{Shuffle ExChange Network CATEgory}

SEN is a unique path it has only a single path between a particular input and output [9]. Shuffle Exchange Network configuration depends on the number of stages, switches and interconnection among the network. The following subsections are organized as follows. Sub-sections review the Shuffle Exchange Network from previous works according to SEN with additional stages, Improved Irregular Augmented Shuffle Multistage Interconnection Network (IIASN), Irregular Modified Alpha Network (ALN), Irregular Augmented Shuffle Network (IASN), Irregular Augmented Shuffle Exchange Network (IASEN) and Generalized Shuffle Exchange Network (GSEN).

\section{A. SEN with Additional Stages}

SEN with additional stage $(\mathrm{SEN}+)$ provide more redundant path for the routing procedure process. It is able to improve the performance in term of reliability performance. Reliability of MIN is used as a measure of system ability to transform information from input to output devices [5]. There are three types of Shuffle Exchange Networks (SEN) from the previous work, which is SEN, SEN with additional stage $(\mathrm{SEN}+)$ and SEN with two additional stages $(\mathrm{SEN}+2)$. Basic SEN is a unique path it has only a single path between a particular input and output [4]. Different from the basic SEN, SEN+ provide two paths between each source and destination and it has $\mathrm{N}$ input and $\mathrm{N}$ output. For $\mathrm{SEN}+2$, it consists of four paths between any pair of input and output. From the results, it can be observed that by adding one additional stage it is more efficient and improved the reliability performance rather than adding with a two stages. It also can be concluded that, by adding two additional stages in the SEN+2 do not increase the reliability of the network since the links complexity leads to higher network system failure. However, there is a solution can be considered by provide a permutation in the middle of stages so the switches will be routing independently and cannot cause the link complexity. It also can increase the reliability since it has multiple path can be used to route the source to destination. With this permutation when the fault occurs in the first stages the routing can be route in multiple paths so it doesn't cause a system failure.

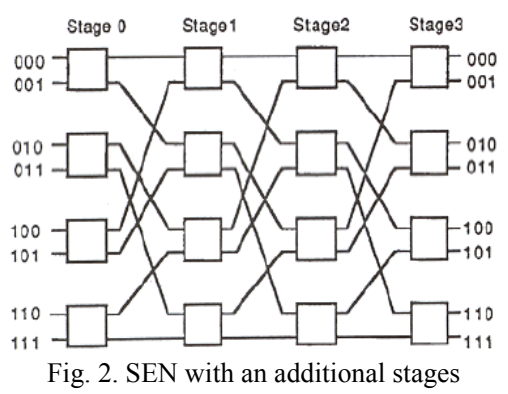

\section{B. Improved Irregular Augmented Shuffle Multistage Interconnection Network (IIASN)}

An Improved Irregular Augmented Shuffle Multistage Interconnection Network (IIASN) is design to provide inter process communication and developed fault tolerant architecture [12]. IIASN has improved path length and cost effectiveness analysis [12]. This type of network provides permutation to route source to destination when both switches in a loop are simultaneously faulty at any stages. From the result it can be observe the permutation passable of IIASN is much better than existing networks such as IASN, FT and MFT. However in this types of network the multiplexer did not offer an alternative way to provide multiple paths if a fault occur in the first stage. For the solution the looping function is the better choice to overcome this problem. By providing looping function in multiplexer so it can create a multiple paths if fault occurs in the first stage.

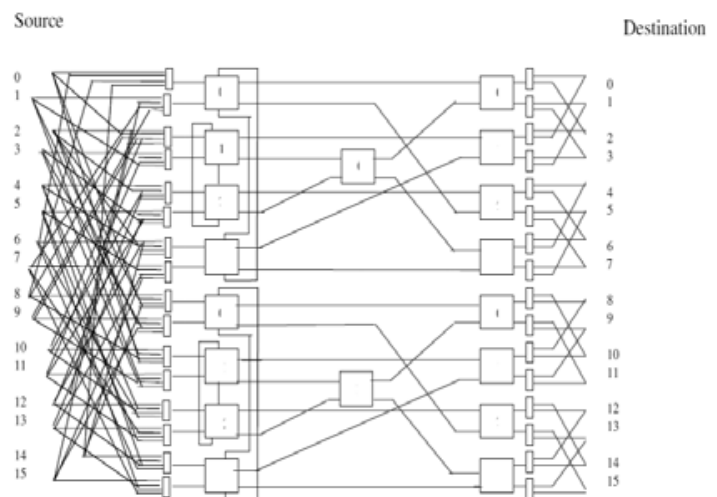

Fig. 3. IIASN MIN of size $\mathrm{N}=16$

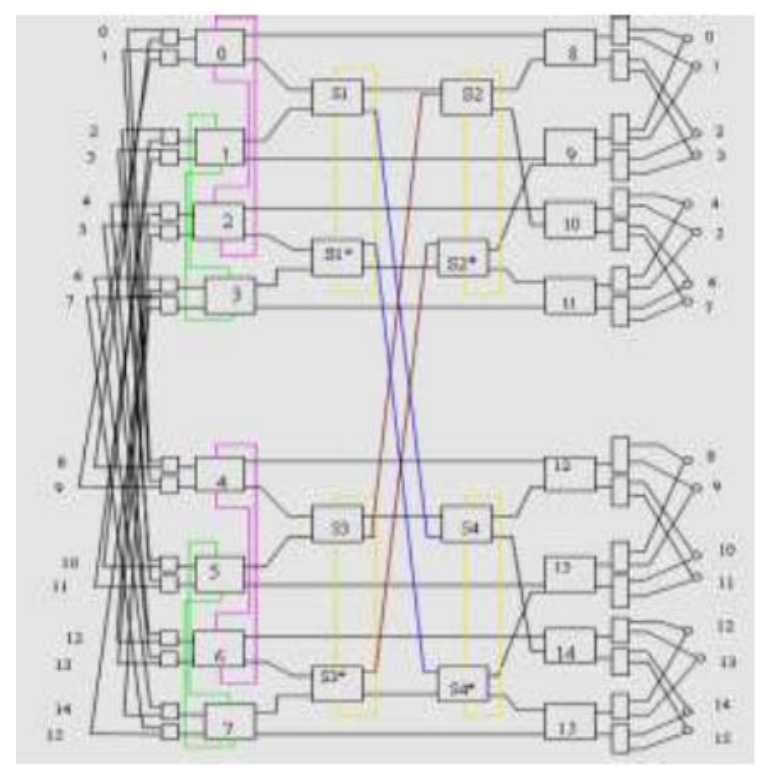

Fig. 4. 16x16 Modified Alpha Network 


\section{Irregular Modified Alpha Network (ALN)}

The Irregular Modified Alpha Network (Modified ALN) has proposed to reduce the cost and latency in the interconnection process [1]. The number of stages in this network is lesser compared to Alpha Network but it can maintain the full access capability at the same time. This network is more reliable because it has auxiliary link to route source to destination when SE in the next stage faulty or busy [1]. This network attains higher values of other important parameters. Modified ALN provide two identical groups of SE which is G0 and G1 when the G0 faulty then it will pass the task to G1 to complete the job, but when all the SEs in the loops in both groups fails, Modified ALN also fail. To solve this problem in the future, it can be done by replacing the loop engaged in the faulty component by using an efficient scheduling.

\section{Irregular Augmented Shuffle Network (IASN)}

An innovative class of irregular fault tolerant multistage interconnection network called Irregular Augmented Shuffle Network (IASN) has been designed and evaluate. The irregular network IASN helps in dropping the latency or delay. IASN has been considered in a way to develop the performance of the network. The recommended IASN networks assure the fault tolerance criteria it can be operate even in existence of assured faults. The existences of the auxiliary links accessible in the network afford an alternate path used for routing procedure, except at the last stage [3]. It has decreased number of stages thus exhibiting reduced latency and enhanced performance. IASN is a single switch fault tolerance. By adding multiple switches it can provide a better network performance. In addition by upgrading, the network environment to the multi switch fault tolerance, it also can improve a better performance for the network.

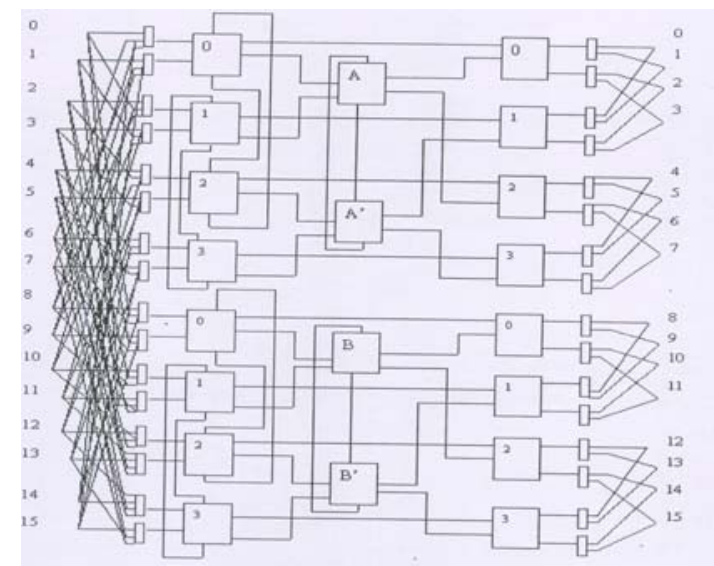

Fig. 5. Irregular Augmented Shuffle Network (IASN)

\section{E. Irregular Augmented Shuffle Exchange Network (IASEN)}

IASEN improved a better fault-tolerance by afford the additional paths among any pair of source destination as contrast to ASEN-2 at the expanse of small extra cost [10]. IASEN is a dynamically re-routable and provides multiple paths of varying lengths between a given source and destination pair. IASEN provide eight alternate paths for routing procedure. Even though it can help to route the switch when the previous path is failure but with many path available it will leads a complexity to the switches. By using permutation and scheduling the path the routing process will become smoothly and also it can increase the performance of network.

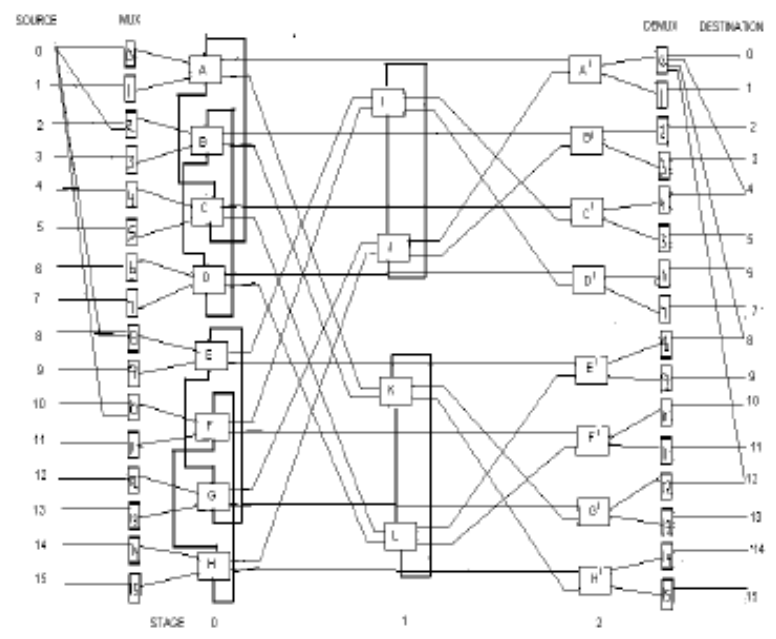

Fig. 6. Irregular Augmented Shuffle Exchange Network 16x16

\section{F. Generalized Shuffle Exchange Network (GSEN)}

All-to-all personalized exchange mostly occurs in parallel and distributed computing applications. An all-to-all communication algorithm is said to be optimal if it has the smallest communication delay [14]. MIN is a better choice for implementing all-to-all personalized exchange due to its shorter communication delay and better scalability. This algorithm working successfully with unique path property provide by Multistage Interconnection Network. In Generalized Shuffle Exchange Network (GSEN) the algorithms cannot be used because GSEN did not provide the unique path property. Optimal algorithm can be considering the all to all personalized exchange algorithm in this GSEN. Optimal algorithm consist of several bounds on $\mathrm{R}(\mathrm{N})$ and $\operatorname{Rsc}(N)$ [14]. Recall that $R(N)$ is the minimum number of network configurations required to fulfill all-to-all communication in an $\mathrm{N} \times \mathrm{N}$ GSEN and Rsc (N)is the minimum number of network configurations required to fulfill all-to-all communication in an N x N GSEN when the stage control technique is assumed [14]. Refer to the previous research, several open problems can be found in Fig. 14 in this paper $\{14]$. In particular, this algorithm conjecture $\mathrm{R}(36)$ $=40, \mathrm{R}(44)=48$. Determining $\mathrm{R}(\mathrm{N})$ for all $\mathrm{N}$ such that $\mathrm{N} \equiv 0$ $(\bmod 4)$ is still an open problem. To overcome this situation by proving the theorem that $\mathrm{R}(36) \geq 40$ is needed.

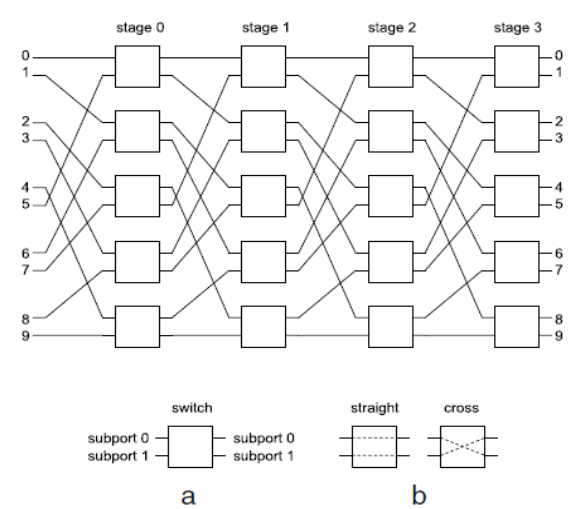

Fig. 7. 10x10 Generalized Shuffle Exchange Network 


\section{COMPARATIVE ANALYSIS}

SEN scheme has considered as cost effectiveness in Multistage Interconnection Network environment. Table 1 shows the comparative analysis for the reviewed schemes. For the comparison, most of the proposed schemes are initially focusing on network performance. SEN with their own scheme has different technique to solve the problem in the Multistage Interconnection with their own behavior. This different technique helped to provide more redundant path to improve the network performance. Besides that, it also helped in term of developing the fault tolerant and reducing the latency.

References :

$$
\begin{aligned}
& 1 \text { - SEN with Additional Stages } \\
& 2 \text { - IIASN } \\
& 3 \text { - Modified ALN } \\
& 4 \text { - IASN } \\
& 5 \text { - IASEN } \\
& 6 \text { - GSEN }
\end{aligned}
$$

\begin{tabular}{|c|c|c|c|c|c|c|}
\hline Criteria & 1 & 2 & 3 & 4 & 5 & 6 \\
\hline Additional Stages & $\sqrt{ }$ & & & & & $\sqrt{ }$ \\
\hline Reduce Stage & & & $\sqrt{ }$ & $\sqrt{ }$ & & \\
\hline Alternative Path & $\sqrt{ }$ & $\sqrt{ }$ & & & $\sqrt{ }$ & \\
\hline Switching Element (SE) & $\sqrt{ }$ & & $\sqrt{ }$ & & $\sqrt{ }$ & \\
\hline Shuffle Exchange Process & $\sqrt{ }$ & $\sqrt{ }$ & $\sqrt{ }$ & $\sqrt{ }$ & $\sqrt{ }$ & $\sqrt{ }$ \\
\hline Groups & & & $\sqrt{ }$ & $\sqrt{ }$ & $\sqrt{ }$ & \\
\hline Multiplexer and Demultiplexer & & $\sqrt{ }$ & $\sqrt{ }$ & $\sqrt{ }$ & $\sqrt{ }$ & \\
\hline Routing Tags & & $\sqrt{ }$ & & $\sqrt{ }$ & $\sqrt{ }$ & $\sqrt{ }$ \\
\hline Routing Procedure & & $\sqrt{ }$ & $\sqrt{ }$ & $\sqrt{ }$ & $\sqrt{ }$ & $\sqrt{ }$ \\
\hline Reliability Analysis & $\sqrt{ }$ & & & $\sqrt{ }$ & & \\
\hline Auxiliary Links & & & $\sqrt{ }$ & & & \\
\hline Fault Tolerance & $\sqrt{ }$ & $\sqrt{ }$ & $\sqrt{ }$ & $\sqrt{ }$ & $\sqrt{ }$ & \\
\hline Latency & & & $\sqrt{ }$ & $\sqrt{ }$ & & \\
\hline Delay & & & & $\sqrt{ }$ & & \\
\hline Cost Effectiveness & & $\sqrt{ }$ & $\sqrt{ }$ & & $\sqrt{ }$ & \\
\hline Permutation & & $\sqrt{ }$ & & & $\sqrt{ }$ & $\sqrt{ }$ \\
\hline Effective Network Size & 8 & 16 & 16 & 16 & 16 & 10 \\
\hline
\end{tabular}

TABLE I: SEN SCHEME ANALYSIS

\begin{tabular}{|c|c|}
\hline Scheme & Challenges \\
\hline $1 \begin{array}{l}\text { SEN+ have the highest } \\
\text { reliability performance }\end{array}$ & $\begin{array}{l}\text { It is noted that by adding two additional } \\
\text { stages in the SEN+2 do not increase the } \\
\text { reliability of the network since the links } \\
\text { complexity leads to higher network system } \\
\text { failure. }\end{array}$ \\
\hline $\begin{array}{l}\text { IIASN has better path } \\
\text { length and permutation } \\
\text { passable compared to } \\
\text { FT and MFT. }\end{array}$ & $\begin{array}{l}\text { The multiplexer did not offer an alternative } \\
\text { way to provide multiple paths if a fault occur } \\
\text { in the first stage. }\end{array}$ \\
\hline $\begin{array}{l}\text { Modified ALN reduced } \\
\text { the number of stages } \\
\text { and reduce latency. }\end{array}$ & $\begin{array}{l}\text { Modified ALN provide two identical groups } \\
\text { of SE which is G0 and G1 when the G0 faulty } \\
\text { then it will pass the task to G1 to complete } \\
\text { the job. But when all the SEs in the loops in } \\
\text { both groups fails, Modified ALN also fail. }\end{array}$ \\
\hline $\begin{array}{l}\text { IASN reduced the } \\
\text { number of stages and } \\
\text { reduce latency. }\end{array}$ & $\begin{array}{l}\text { IASN is a single switch fault tolerance with a } \\
\text { lesser network performance }\end{array}$ \\
\hline $\begin{array}{l}\text { IASEN provide much } \\
\text { more fault tolerance } \\
\text { than ASEN-2. }\end{array}$ & $\begin{array}{l}\text { IASEN provide } 8 \text { alternate paths for routing } \\
\text { procedure. Even though it can help to route } \\
\text { the switch when the previous path is failure }\end{array}$ \\
\hline
\end{tabular}

However, with the different techniques proposed there

\begin{tabular}{|c|c|c|}
\hline & & $\begin{array}{l}\text { but with may path available it leads a } \\
\text { complexity to the switches. }\end{array}$ \\
\hline 6 & $\begin{array}{l}\text { Optimal algorithm } \\
\text { fulfill all to all } \\
\text { communication in } \\
\text { GSEN configuration. }\end{array}$ & $\begin{array}{l}\text { Several open problems can be found in } \\
\text { GSEN. In particular, this algorithm } \\
\text { conjecture } R(36)=40, R(44)=48 \text {. } \\
\text { Determining } R(N) \text { for all } N \text { such that } N \equiv 0 \\
(\bmod 4) \text { is still an open problem. }\end{array}$ \\
\hline
\end{tabular}
also have challenges for each technique. The comparison of reviewed schemes and their challenges was reviewed below in Table II.

TABLE II: SEN ISSUES AND CHALLENGES

\section{CONCLUSION}

Shuffle exchange network has been proposed as a popular architecture for MINs. In this paper, we investigated six different schemes of Shuffle Exchange Network in Multistage Interconnection Network namely SEN with additional stages, Improved Irregular Augmented Shuffle Multistage Interconnection Network (IIASN), Irregular Modified Alpha Network (ALN), Irregular Augmented Shuffle Network (IASN), Irregular Augmented Shuffle Exchange Network (IASEN) and Generalized Shuffle Exchange Network (GSEN). This paper review the scheme provided from the SEN in MINs environment. It can be observed from the analysis that, SEN scheme can provide a more redundant path by adding the stages. It also can developed the fault tolerant and reduce the latency of the network by provide an auxiliary links. Dynamically, most of the researchers are consider to using the SEN scheme in MINS architecture since it be capable to reducing the cost in MINs architecture. However these schemes also have their own challenges. Some techniques such as adding some permutation or routing procedure can help to overcome the problem in MINs. From this review, it can be conclude that the technique use is important to describe the network performance measurement. Other than that another issues that related to the network performance is the sizes of network using in that networks. For an illustration, if the network used the large network size it can cause a complexity in the network. An addition by using a large network size also can increase the cost and can cause a system failure. In general, MIN is suitable to apply in different types of network. It can help reducing the cost and increase the network performance. Future research will focus on some techniques that alter the topology of a MIN for a better fault tolerance. Besides that, link failures can also be taken into consideration for future investigation.

\section{ACKNOWLEDGMENT}

This work was supported by the Research University Grant Scheme (RUGS), Universiti Putra Malaysia (RUGS Number: 05/01/07/0180RU).

\section{REFERENCES}

[1] Amardeep Gupta and Dr. P K Bansal 2010. Fault Tolerant Irregular Modified Alpha Network and Evaluation of Performances Parameter, International Journal of Computer Applications, Vol (4). pp. 9-13.

[2] Dietmar Tutsch and Gunter Hommel 2003. Multilayer Multistage Interconnection Networks, Proceedings of 2003 design, analysis, and simulation of distributed systems (DASD'03). Orlando, USA, pp.155-162.

[3] Harsh Sadawarti,Bansal P.K. 2007. Fault Tolerant Irregular Augmented Shuffle Network, Proceedings of the 2007 WSEAS International Conference on Computer Engineering and Applications. Gold Coast, Australia, January pp.17-19.

[4] Indra Gunawan 2008. Reliability Analysis of Shuffle Exchange Networks System, Reliability Engineering and System Safety 93, pp. 
271-276.

[5] Indra Gunawan and Nasser S. Fard 2007. Reliability Evaluation of Optical Multistage Interconnection, Proceeding ICTON-MW, pp. 1-4.

[6] Indra Gunawan and Nasser S. Fard 2002, Reliability Bounds for Large Multistage Interconnection Networks, Conference on Applied Parallel Computing (PARA'02), Espoo, Finland , pp. 507-514

[7] Indra Gunawan., Palaniappan, S., and Lim, C. S. 2004, "Extra-Stage Cube Network Reliability Estimation Using Stratified Sampling Monte Carlo Method", $3^{\text {rd }}$ Technical Postgraduate Symposium, University of Malaya, Malaysia, pp. 13-18.

[8] Indra Gunawan, 2004, Redundant Paths in a Gamma Network, $12^{\text {th }}$ INFORMS/Applied Probability Society, Beijing, China, pp. 588-594.

[9] Indra Gunawan and Nasser S. Fard 2007. Multistage Interconnection Networks Reliability, Citeseer.pp. 1-6.

[10] Lee Weng Kett 2004.Shuffle Exchange Network with an Additional Stage (SEN+) Reliability Simulation, Master Thesis, Malaysia University of Science and Technology.

[11] Rinkle Aggarwal and Dr. Lakhwinder Kaur 2010. Fault Tolerance and Permutation Analysis of ASEN and its Variant, International Journal of Computer Science and Information Technologies, Vol (1): pp. 24-32.

[12] Ragha Vendra, and Anujan Varma 1987. Rearrangeability of The Five Stage Shuffle Exchange Network for $\mathrm{N}=8$, IEEE Transaction on Communications, Vol (3) Issue (8): pp. 808-812.

[13] Sandeep Sharma, Dr. K.S.Kahlon, Dr. P.K.Bansal, Dr.and Kawaljeet Singh 2007. Improved Irregular Augmented Shuffle Multistage Interconnection Networks, International Journal of Computer Science and Security, Vol (2) Issue (3) pp. 28-33.

[14] Siu-Cheung Chau, Tiehong Xiao and Ada Wai-Chee Fu 2008. Routing and Scheduling for a Novel Optical Multistage Interconnection Network, J.C. Cunha and P.D. Medeiros (Eds.), Euro-Par, LNCS, pp. 984-993.

[15] Well Y. Chou, and Chiuyuan Chen 2010, All-to-all Personalized Exchange In Generalized Shuffle Exchange Network, Theoretical Computer Science, Vol (411), Issues (16-18), 28 March 2010, Pages 1669-1684.
[16] R Mahajan, and R Vig. 2008, Performance and reliability analysis of new fault-tolerant advance omega network, WSEAS Transactions on Computers. Vol (7) 1280-1290.

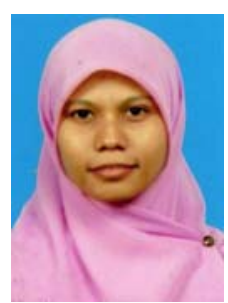

Nur Arzilawati Md Yunus is a Master degree student of Department of Communication Technology and Network at Universiti Putra Malaysia. She received Bachelor of Computer Science major in Computer Network from University Putra Malaysia in 2010.

She currently continued her study in Master of Science focusing on Computer Network at University Putra Malaysia. She is a member of the International Association of Computer Science and Information Technology Press (IACSIT). Her research interests are focusing on shuffle exchange network, multistage interconnection networks, optical switching, parallel computing and routing algorithm.

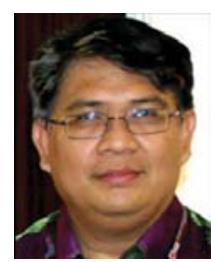

Mohamed Othman is a Professor of Department of Communication Technology and Network at Universiti Putra Malaysia. He also appointed as a Deputy Dean for Research, Post Graduate, and Finance.

$\mathrm{He}$ is a member of the Institute of Electrical and Electronics Engineers (IEEE) Computer Society (Malaysian Section), the Institute of Electronics, Information and Communication Engineers (IEICE) of Japan, Malaysian National Computer Confederation and life membership for Malaysian Mathematical Society. He is also a member of the International Association of Engineers (IAENG) and the World Internet Society (ISOC).He has expertise in several areas of computer science such as parallel and distributed algorithms, grid computing, high-speed computer network and scientific computing.

He received his $\mathrm{PhD}$ in Computer Science from the Department of Industrial Computing, Universiti Kebangsaan Malaysia with distinction (Best PhD Thesis in 2000 awarded by Sime Darby Malaysia and Malaysian Mathematical Science Society) in 1999. 